\title{
Scalar wave propagation in topological black hole backgrounds
}

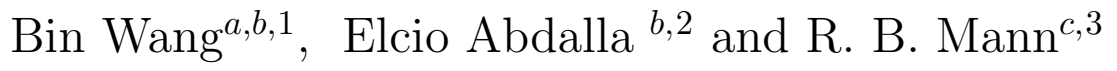 \\ ${ }^{a}$ Department of Physics, Fudan University, Shanghai 200433, P. R. China \\ ${ }^{b}$ Instituto De Fisica, Universidade De Sao Paulo, C.P.66.318, CEP 05315-970, \\ Sao Paulo, Brazil \\ ${ }^{c}$ Department of Physics, University of Waterloo, Waterloo, Ontario, Canada \\ N2L 3G1
}

PACS number(s): 04.30.Nk, 04.70.Bw

\begin{abstract}
We consider the evolution of a scalar field coupled to curvature in topological black hole spacetimes. We solve numerically the scalar wave equation with different curvature-coupling constant $\xi$ and show that a rich spectrum of wave propagation is revealed when $\xi$ is introduced. Relations between quasinormal modes and the size of different topological black holes have also been investigated.
\end{abstract}

\footnotetext{
${ }^{1}$ e-mail:binwang@fma.if.usp.br

${ }^{2}$ e-mail:eabdalla@fma.if.usp.br

${ }^{3}$ e-mail:rbmann@sciborg.uwaterloo.ca
} 


\section{Introduction}

Radiative wave propagation in spacetimes containing a black hole has been a subject of investigation for a number of years. It is well known that after an initial pulse, the waveform undergoes quasinormal ringing outside the black hole. The frequencies of the "ring" are independent of the processes which give rise to these oscillations, and instead are directly connected to the parameters of the black hole. It is generally believed that these quasinormal frequencies carry a unique fingerprint that leads to direct identification of a black hole, thereby allowing confirmation of their existence through anticipated gravitational wave observation in the near future [1].

A great deal of effort has been devoted to the study of the quasinormal modes associated with black holes. Most of these studies were concerned with black holes immersed in an asymptotically flat spacetime. The perturbations of Schwarzschild and Reissner-Nordstrom (RN) black holes can be reduced to sim-

ple wave equations which have been examined extensively [2] [3] [4. However, for nonspherical black holes one has to solve coupled wave equations for the radial part and angular part, respectively. For this reason the nonspherical case has been studied less thoroughly, although there has recently been progress along these lines [5] [6] [7]. In asymptotically flat black hole backgrounds radiative dynamics always proceeds in the same three stages: initial impulse, quasinormal ringing and inverse power-law relaxation.

Increasing interest has been expressed in non-asymptotically flat spacetimes. For a black hole is immersed in an expanding universe, it has been shown that while the first two stages of radiative dynamics are not affected by the different conditions at infinity, the third one changes [8] 9] [10]. At late times the field decays exponentially, in contrast to an inverse power law as in the asymptotically flat case. More thorough exploration of the quasinormal modes of scalar fields coupled to the curvature have shown that the field's behaviour strongly depends 
on the value of the curvature coupling constant.

The first study of the quasinormal ringing for conformally coupled scalar waves in AdS space was performed by Chan and Mann [1] [12]. Interest in the asymptotically AdS case has since grown considerably, motivated by the recent discovery of the AdS/CFT correspondence. In this context one expects that the quasinormal frequencies of AdS black holes have a direct interpretation in terms of the dual conformal field theory (CFT) on the boundary of the asymptotically AdS spacetime. Recently, Horowitz and Hubeney [13] considered scalar quasinormal modes on the background of Schwarzschild AdS black holes in four, five and seven dimensions. They claimed that for large AdS black holes both the real and imaginary parts of the quasinormal frequencies scale linearly with the black hole temperature. However for small AdS black holes they found a departure from this behaviour. This was further confirmed by the object picture obtained in ref. [14]. These investigations were further generalized to Reissner-Nordstrom (RN) AdS black holes, which provide a broader parameter space than uncharged Schwarzchild AdS (SAdS) black holes [15] [16]. Unlike the SAdS case, the quasinormal frequencies do not scale linearly with the black hole temperature, and the approach to thermal equilibrium in the CFT was more rapid as the charge on the black hole increased. Further extensions in asymptotically AdS spacetimes have included investigations of quasinormal modes due to electromagnetic and gravitational perturbations [17], and of nonspherical Kerr-Newmann AdS black hole backgrounds [18].

So far all investigations on wave dynamics in AdS spacetimes have been limited to fields that are either conformally coupled [11] [12] or minimally coupled to curvature [13, 14, 15, 16, 17]. In de Sitter spacetimes the behaviour of the scalar field has considerable dependence on the value of the curvature-coupling constant, and we are likewise motivated to study the dependence of scalar quasinormal modes on the curvature-coupling constant in AdS spacetimes, a study we carry out in this paper. We generalize the study to include topological black holes, 
whose quasinormal ringing properties have only been examined in the conformally coupled case [12]. Topological black hole spacetimes are all asymptotically AdS spacetimes, and have black hole event horizons which are compact two-surfaces of arbitrary genus [19], with genus zero being the SAdS case. We solve numerically the wave equations with a general non-minimal coupling of the scalar field to the spacetime curvature. We find that the number of possible different behaviours of quasinormal modes for scalar field is significantly enhanced as the non-minimal coupling parameter $\xi$ is varied.

Our paper is organized as follows: in section 2 we review the basic structures of topological black hole spacetimes and derive the equation governing the scalar test field coupled to curvature. In the next section we analyze behaviours of scalar waves in spherical (genus $g=0)$, toroidal $(g=1)$ and higher genus $(g \geq 2)$ black hole backgrounds and their dependence on curvature coupling constants. Conclusions and a discussion of resutls are included in the last section.

\section{Equations and numerical methods}

The spacetime of an uncharged topological black hole of arbitrary genus $g \geq 0$ has the general form [19] [1]

$$
\begin{gathered}
\mathrm{d} s^{2}=-N_{g}(r) \mathrm{d} t^{2}+N_{g}(r)^{-1} \mathrm{~d} r^{2}+r^{2} \mathrm{~d} \Omega^{2}, \\
N_{g}(r)=r^{2} / l^{2}-\epsilon(g-1)-2 M / r
\end{gathered}
$$

where

$$
\epsilon(g-1)=[\Theta(g-1)-\Theta(1-g)]= \begin{cases}-1 & g=0 \\ 0 & g=1 \\ 1 & g>1\end{cases}
$$




$$
\mathrm{d} \Omega^{2}= \begin{cases}\mathrm{d} \theta^{2}+\sin ^{2} \theta \mathrm{d} \phi^{2} & g=0 \\ \mathrm{~d} \theta^{2}+\mathrm{d} \phi^{2} & g=1 \\ \mathrm{~d} \theta^{2}+\sinh ^{2} \theta \mathrm{d} \phi^{2} & g>1\end{cases}
$$

and $l=\sqrt{3 /|\Lambda|}$.

When $g=0$ the metric is that of Schwarzschild AdS spacetime. When $g=1$, the coordinates $\theta$ and $\phi$ describe a flat space and the metric corresponds to a toroidal spacetime. When $g \geq 2$, the coordinate $(\theta, \phi)$ are coordinates of a hyperbolic space and the spacetime is referred to as a topological black hole. Note that the topology of the event horizon matches the topology out to infinity.

We consider a massless scalar field $\Phi$ in spacetime (1) obeying the wave equation

$$
(\square-\xi R) \Phi=0
$$

where $\square=g^{\alpha \beta} \nabla_{\alpha} \nabla_{\beta}$ is the d'Alembertian operator, $R=-4|\Lambda|$ the Ricci scalar, and $\xi$ a tunable curvature coupling constant. If we decompose the scalar field according to

$$
\Phi=\sum \frac{1}{r} \psi(t, r) Y(\theta, \phi)
$$

then each wave function $\psi(r)$ satisfies the equation

$$
-\frac{\partial^{2} \psi}{\partial t^{2}}+\frac{\partial^{2} \psi}{\partial X_{g}^{2}}=V \psi
$$

where

$$
V=N_{g}\left[\frac{2}{l^{2}}(1-6 \xi)+\frac{2 M}{r^{3}}\right]
$$

and $X_{g}=\int N_{g}{ }^{-1} d r$ is the tortoise coordinate.

To compare the wave behaviour for black holes of different topologies, we follow ref. [11] and perform a rescaling $r=r_{+} z$ so that the event horizon is at unit dimensionless distance. Adopting this dimensionless variable $z$, we obtain

$$
\tilde{N}_{g}=l^{2} N_{g} / r_{+}^{2}=\frac{z-1}{z}\left(z^{2}+z+1-\frac{l^{2}}{r_{+}^{2}} \epsilon(g-1)\right) .
$$


After rescaling the scalar wave equation (7) becomes

$$
-\frac{\partial^{2} \tilde{\psi}}{\partial \tilde{t}^{2}}+\frac{\partial^{2} \tilde{\psi}}{\partial \tilde{X}_{g}^{2}}=\tilde{V} \tilde{\psi}
$$

where

$$
\begin{aligned}
\tilde{t}= & \frac{r_{+}}{l^{2}} t \\
\tilde{X}_{g}= & \frac{r_{+}}{l^{2}} X_{g} \\
\tilde{V}= & {\left[1-3 \beta^{2} \epsilon(g-1)\right] \frac{z-1}{z^{4}}\left[z^{2}+z+1-3 \beta^{2} \epsilon(g-1)\right] } \\
& +2 \chi \frac{z-1}{z}\left[z^{2}+z+1-3 \beta^{2} \epsilon(g-1)\right] \text { and } \chi=1-6 \xi \\
\beta= & \frac{l}{\sqrt{3} r_{+}}>0
\end{aligned}
$$

For different black holes with different topologies, the rescaled tortoise coordinates $\tilde{X}_{g}$ are 11

$$
\begin{aligned}
\tilde{X}_{g=0}= & \frac{1}{3\left(1+\beta^{2}\right)}\left[\ln \frac{z-1}{\sqrt{z^{2}+1+1+3 \beta^{2}}}+\frac{\sqrt{3}\left(1+2 \beta^{2}\right)}{\sqrt{1+4 \beta^{2}}} \arctan \left(\frac{2 z+1}{\sqrt{3+12 \beta^{2}}}\right)\right] \\
\tilde{X}_{g=1}= & \frac{1}{3} \ln \frac{z-1}{\sqrt{z^{2}+z+1}}+\frac{1}{3} \arctan \left(\frac{2 z+1}{\sqrt{3}}\right) \\
\tilde{X}_{g \geq 2}= & \frac{1}{3\left(1-\beta^{2}\right)}\left[\ln \frac{z-1}{\sqrt{z^{2}+1+1-3 \beta^{2}}}+\frac{\sqrt{3}\left(1-2 \beta^{2}\right)}{\sqrt{1-4 \beta^{2}}} \arctan \left(\frac{2 z+1}{\sqrt{3-12 \beta^{2}}}\right)\right] \quad(0<\beta<1 / 2) \\
\tilde{X}_{g \geq 2}= & \frac{4}{9} \ln \frac{z-1}{z+1 / 2}-\frac{2}{3(2 z+1)} \quad(\beta=1 / 2) \\
\tilde{X}_{g \geq 2}= & \frac{1}{3\left(1-\beta^{2}\right)}\left[\ln \frac{z-1}{\sqrt{z^{2}+1+1-3 \beta^{2}}}\right. \\
& \left.+\frac{\sqrt{3}\left(1-2 \beta^{2}\right)}{\sqrt{4 \beta^{2}-1}} \ln \frac{2 z+1-\sqrt{3} \sqrt{4 \beta^{2}-1}}{2 z+1+\sqrt{3} \sqrt{4 \beta^{2}-1}}\right] \quad(1 / 2<\beta<1) \\
\tilde{X}_{g \geq 2}= & \frac{2}{9} \ln \frac{z-1}{z+2}-\frac{1}{3(z-1)} \quad(\beta=1)
\end{aligned}
$$

We see that for the toroidal topology the rescaled tortoise coordinate is independent of the parameter $\beta$. For the higher genus cases, $\beta$ has a range $0<\beta \leq 1$. $\beta>1$ corresponds to the naked singularity case [11]. The black hole mass can be expressed as $\sqrt{3}(M / l)=1 / \beta^{3}-3 / \beta$ for $g \geq 2$ case. There are two qualitatively 
different parts of $\beta$, namely $0<\beta<1 / \sqrt{3}$ and $1 / \sqrt{3}<\beta<1$, which correspond to two different topological black holes with positive mass and negative mass [20], respectively. $\beta=1 / \sqrt{3}$ corresponds to the zero mass topological black hole.

Using the null coordinates $u=\tilde{t}-\tilde{X}_{g}$ and $v=\tilde{t}+\tilde{X}_{g},(10)$ can be recast as

$$
-4 \frac{\partial^{2}}{\partial u \partial v} \tilde{\psi}(u, v)=\tilde{V}(z) \tilde{\psi}(u, v)
$$

in which $z$ is determined by inverting the relation $\tilde{X}_{g}(z)=(v-u) / 2$.

The two-dimensional wave equation (16) can be integrated numerically, using for example the finite difference method suggested in [3]. Using Taylor's theorem, it is discretized as

$$
\tilde{\psi}_{N}=\tilde{\psi}_{E}+\tilde{\psi}_{W}-\tilde{\psi}_{S}-\delta u \delta v \tilde{V}\left(\frac{v_{N}+v_{W}-u_{N}-u_{E}}{4}\right) \frac{\tilde{\psi}_{W}+\tilde{\psi}_{E}}{8}+O\left(\epsilon^{4}\right)
$$

where the points $N, S, E$ and $W$ form a null rectangle with relative positions as: $N:(u+\delta u, v+\delta v), W:(u+\delta u, v), E:(u, v+\delta v)$ and $S:(u, v) . \epsilon$ is an overall grid scalar factor, so that $\delta u \sim \epsilon \sim \delta v$. Considering that the behaviour of the wave function is not sensitive to the choice of initial data, we set $\tilde{\psi}\left(u, v=v_{0}\right)=0$ and use a Gaussian pulse as an initial perturbation, centered on $v_{c}$ and with width $\sigma$ on $u=u_{0}$ as

$$
\tilde{\psi}\left(u=u_{0}, v\right)=\exp \left[-\frac{\left(v-v_{c}\right)^{2}}{2 \sigma^{2}}\right]
$$

The inversion of the relation $\tilde{X}_{g}(z)$ needed in the evaluation of the potential $\tilde{V}(z)$ is the most tedious part of the computation.

After the integration is completed, the value $\tilde{\psi}\left(u_{\max }, v\right)$ is extracted, where $u_{\max }$ is the maximum value of $u$ on the numerical grid. Taking sufficiently large $u_{\max }, \tilde{\psi}\left(u_{\max }, v\right)$ represents a good approximation for the wave function at the event horizon. Since it has been shown that wave behaviour is the same near or far from the event horizon [3, 12], we will study the dependence of $\tilde{\psi}\left(u_{\max }, v\right)$ on the genus $g$ non-minimal coupling constant $\xi$. 


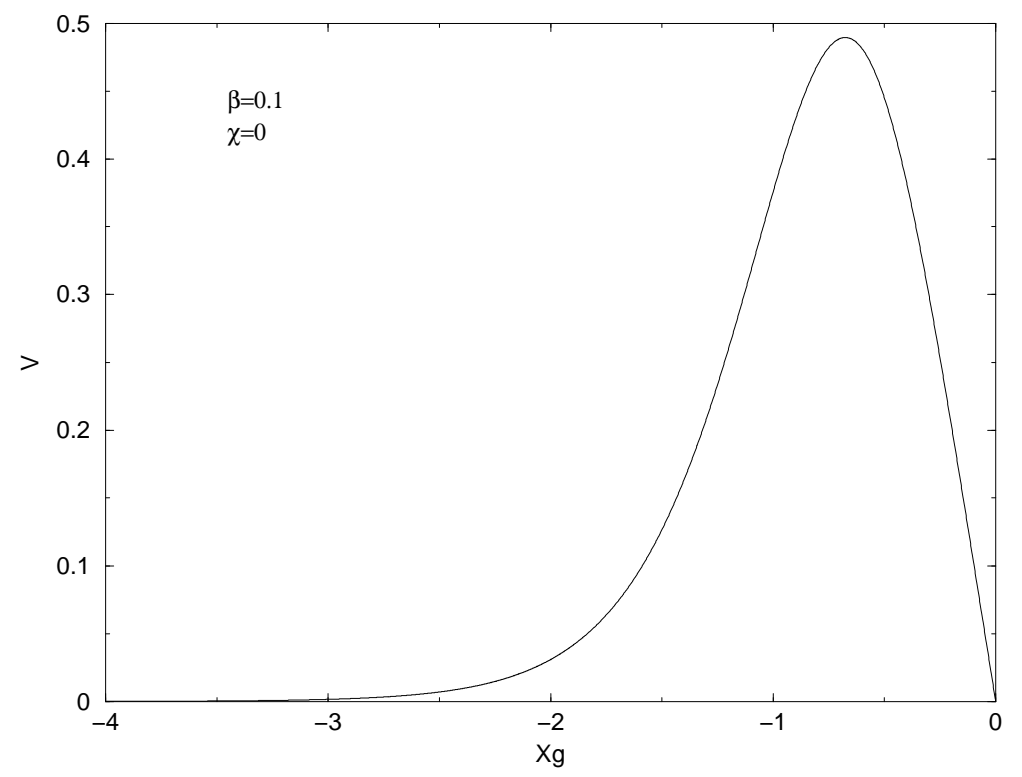

Figure 1: Potential behavior for $\xi=1 / 6$

\section{$3 \quad$ Numerical results}

We now report on the results of our numerical simulations of evolving a massless non-minimally coupled scalar field on different topological black hole spacetimes. In particulars, we will focus on the dependence of the wave behaviour on the coupling constant $\xi$. We will show that this dependence is quite different from that found in de Sitter spacetime [10]. We will also consider the dependence of wave propagation on the parameter $\beta$. For the $g=0$ SAdS case, our results are consistent with that of refs. [13, 14 for either large or small holes. For higher genus holes, we discover some new properties. 


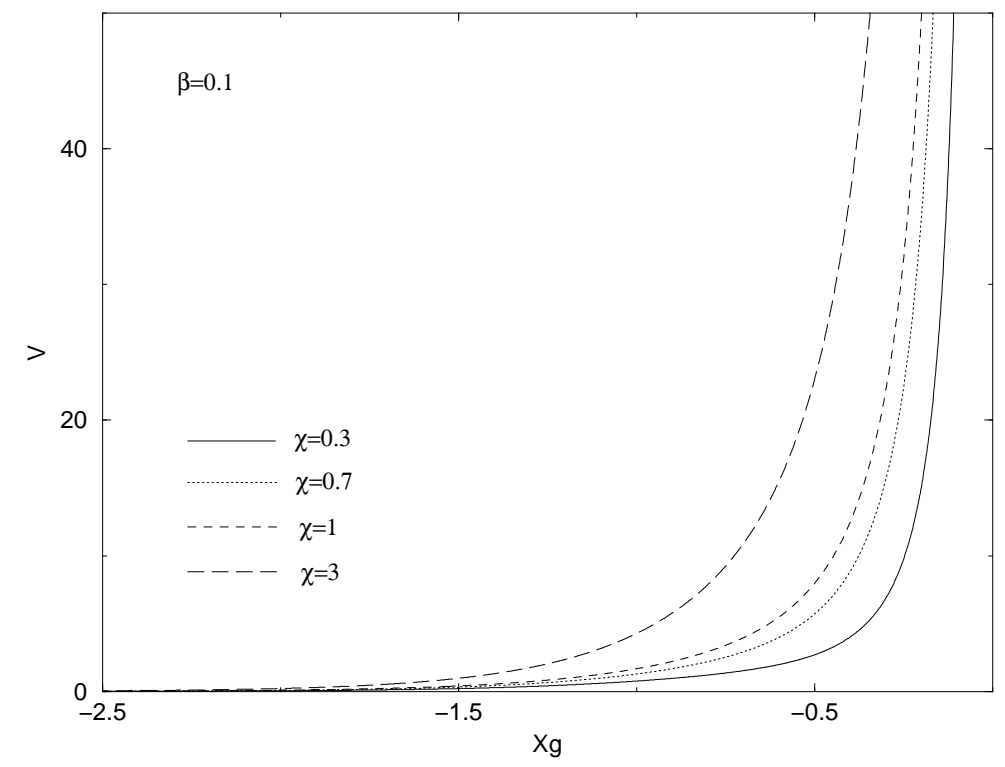

Figure 2: Potential behavior for $\xi<1 / 6$

\subsection{Schwarzschild AdS black hole $(g=0)$ background}

In the $g=0$ or Schwarzschild AdS case, numerical solutions to wave equation of scalar field conformally coupled [11, 12] and minimally coupled to spacetime curvature [13, 14] have been obtained. It was found that the radiative tails associated with a massless scalar wave propagation have an oscillatory exponential decay. This falloff behaviour depends neither on the observation point nor on the initial pulse.

In [10], a scalar non-minimally coupled to curvature was discovered to have a rich spectrum of late-time behaviour in Schwarzschild de Sitter spacetime. We consider in this section the analogous in SAdS spacetimes. It is clear from eq. (13) that the curvature-coupling constant $\xi=\frac{(1-\chi)}{6}$ plays an important role in determining the behaviour of the effective potential $\tilde{V}$. For the scalar field 


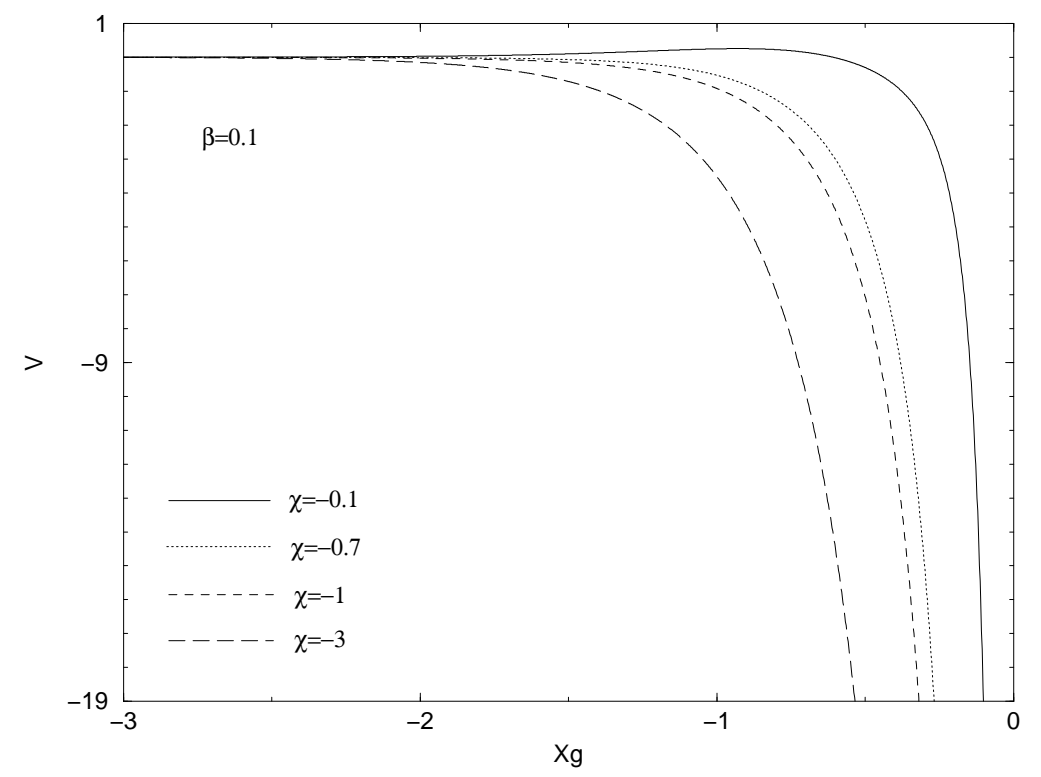

Figure 3: Potential behavior for $\xi>1 / 6$

conformally coupled to curvature $\xi=1 / 6(\chi=0)$, and the graph of this potential function is given in fig. 1 . For $\xi<1 / 6(\chi>0)$, the behaviour of the potential is shown in Fig.2, whereas fig. 3 is the potential for $\xi>1 / 6(\chi<0)$. The dependence of scalar wave propagation on the form of the potential has been noted previously in the asymptotically flat case 21.

Figs. 10 display the quasinormal ringing of the scalar field for several values of $\chi(\xi)$ with fixed $\beta$. For conformally coupled scalar field $(\xi=1 / 6)$, our result coincides with that obtained in [11]. For $\xi \leq 1 / 6(\chi \geq 0)$, we find that both the real and imaginary parts of the quasinormal frequencies increase as $\chi$ increases. This behaviour remains the same as $\beta$ is varied, and differs substantively from that observed in de Sitter spacetime. Since the quasinormal frequencies of black holes in AdS spacetimes have a direct interpretation in terms of the dual CFT, according to the AdS/CFT correspondence the more weakly the scalar field is 

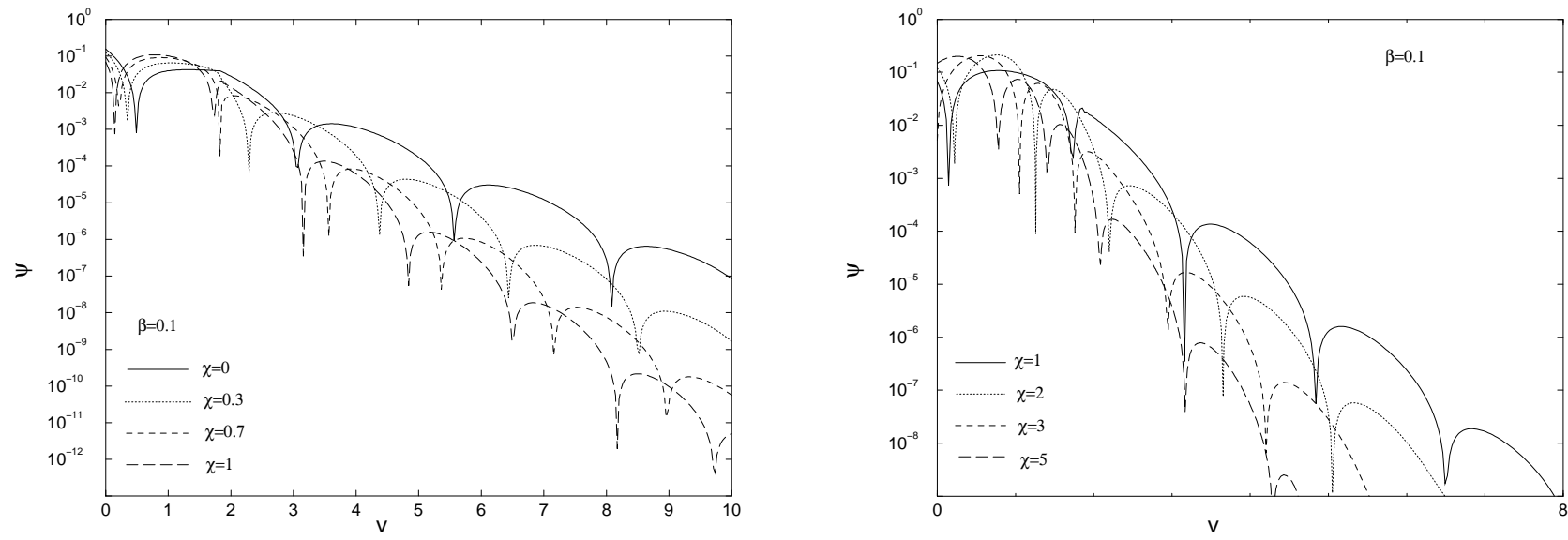

Figure 4: Wave propagation of scalar field for $\xi \leq 1 / 6$ with $\beta=0.1$

non-minimally coupled to the curvature, the faster the thermal state in the CFT settles down to thermal equilibrium.

For $\xi>1 / 6$, the wave propagation amplifies instead of decays outside the black hole. This behaviour can be attributed to the negative infinite potential shown in fig. 3, which implies that the wave outside the black hole gains energy from the spacetime. The close relationship between the varying forms of the potential and the rich spectrum of $\xi$-dependent wave propagation is commensurate with the arguments of Ching et. al.'s [21].

Fig.8 demonstrates the behaviour of the field with increasing $\beta$ from 0.4 to 1 for $\chi=0(\xi=1 / 6)$ in the SAdS black hole background: as $\beta$ increases, the frequency of quasinormal ringing increases and the rate of decay slows. This qualitative behaviour persists at other values of $\chi$. From eq. (14) we see that increasing $\beta$ corresponds to a decreasing black hole size according to the definition given in 13. This stands in apparent contrast to that previously observed for intermediate to small black holes, for which quasinormal modes decay slower 


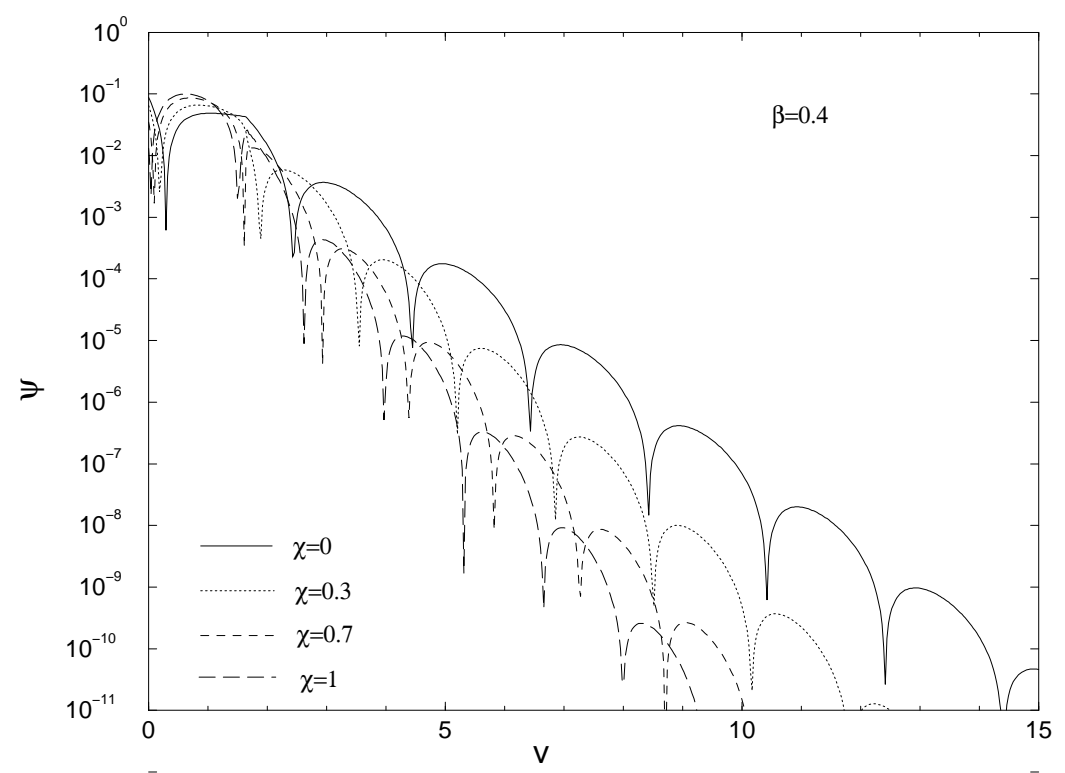

Figure 5: Wave propagation of scalar field for $\xi \leq 1 / 6$ with $\beta=0.4$

for smaller AdS black holes whilst the ringing frequency remains nearly the same [13, 14]. This apparent difference is due to the coordinate transformations (11, 12) we employ, which render our time scale different from that used in refs. [13, 14. Considering (11), the relationship between our frequency $\tilde{\omega}$ and the frequency $\omega$ used in refs. 13, 14, is

$$
\tilde{\omega}=\frac{l^{2}}{r_{+}} \omega .
$$

From (19) we see that an increasing real part of the frequency $\tilde{\omega}_{R}$ corresponds to a nearly constant value of $\omega_{R}$ as the black hole size decreases, as illustrated in fig.8. Likewise, the imaginary part $\tilde{\omega}_{I}$ of the frequency will decrease as $\omega_{I}$ decreases, yielding commensurate decay rates.

For big black holes $\left(r_{+}\right.$large, $\beta$ very small), we have investigated $\beta=0.01,0.005,0.001$ etc. using our rescaled coordinates. We find no difference in wave propagation for these different values of $\beta$. From eq.(19), this corresponds to an increase of 


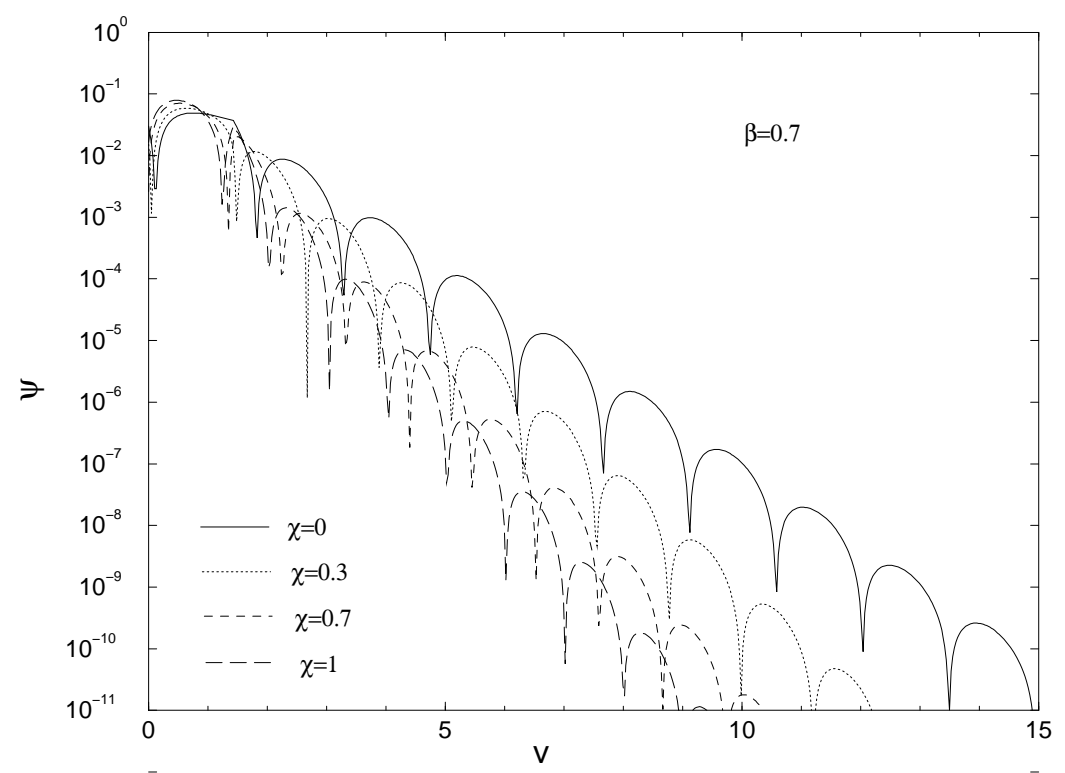

Figure 6: Wave propagation of scalar field for $\xi \leq 1 / 6$ with $\beta=0.7$

both $\omega_{R}$ and $\omega_{I}$ for increasing black hole size, in agreement with the results of large black holes argued in [13]. Throughout, we find that the $\beta$-dependence of the decay rates and frequencies is qualitatively unchanged for different values of $\xi$

\subsection{Toroidal black hole $(g=1)$ background}

Now we proceed to discuss the wave propagation in toroidal black hole background. From (14) and (16) we note that the potential and tortoise coordinate are independent of $\beta$. Consequently the scalar wave behaves the same outside the toroidal black hole for different values of $M / l$. The $\xi$-dependence of the potential for toroidal black holes is qualitatively the same as for the SAdS case, shown in figs.1. 3 . 

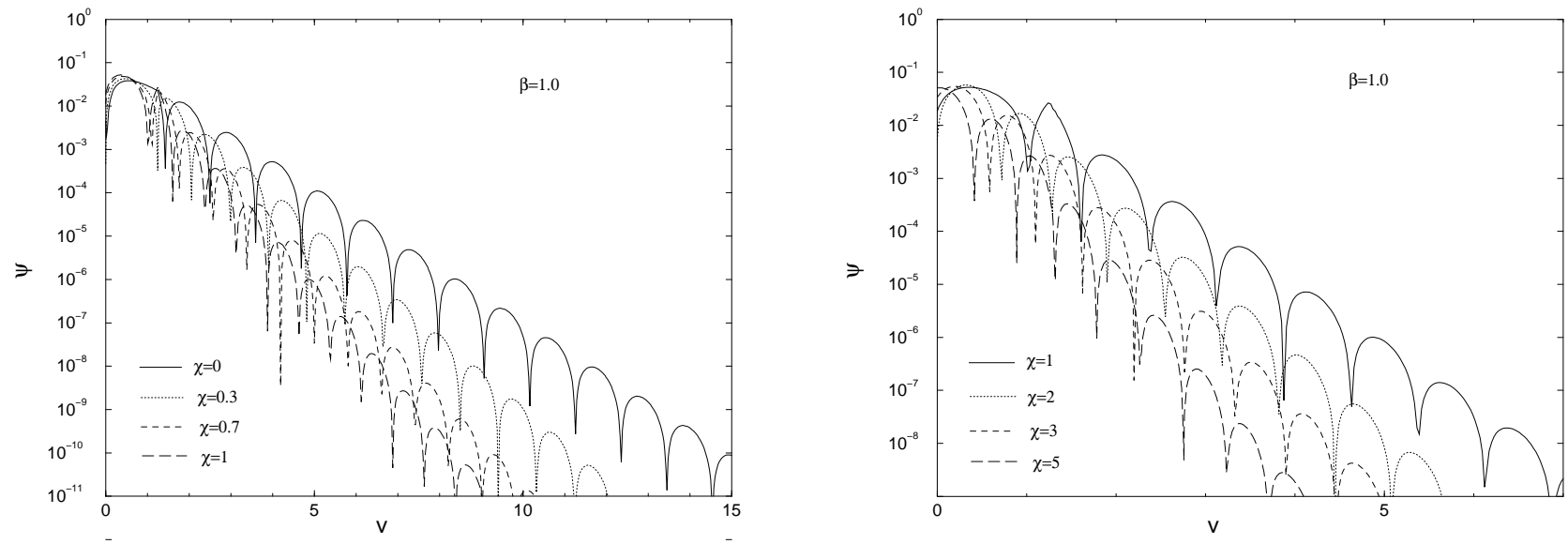

Figure 7: Wave propagation of scalar field for $\xi \leq 1 / 6$ with $\beta=1.0$

Results displayed in fig.9 illustrate the falloff behaviour for $\chi \geq 0(\xi \leq 1 / 6)$ for toroidal holes. It is qualitatively the same as the SAdS case, although quantitatively the decay rate is larger and the wave oscillates with higher frequency. In the context of the AdS/CFT correspondence, any thermal perturbation returns to equilibrium more quickly outside a toroidal hole as $\xi$ decreases relative to the SAdS case. For $\chi<0(\xi>1 / 6)$, we again find amplification modes.

Similar wave behaviour shown for toroidal holes to Schwarzschild AdS holes can be attributed to their similar potential properties. When one considers the argument of Ching et al [21], this similarity is not surprising.

\subsection{Higher genus topological black holes $(g \geq 2)$}

When the genus $g \geq 2$, the geometry of the topological black hole depends on the parameter $\beta$ which runs from 0 to 1 . There are two distinct regions of $\beta$ corresponding to qualitatively different black hole structures. If $0 \leq \beta<1 / \sqrt{3}$, the black hole has positive mass, whereas if $1 / \sqrt{3}<\beta \leq 1$, the black hole has 


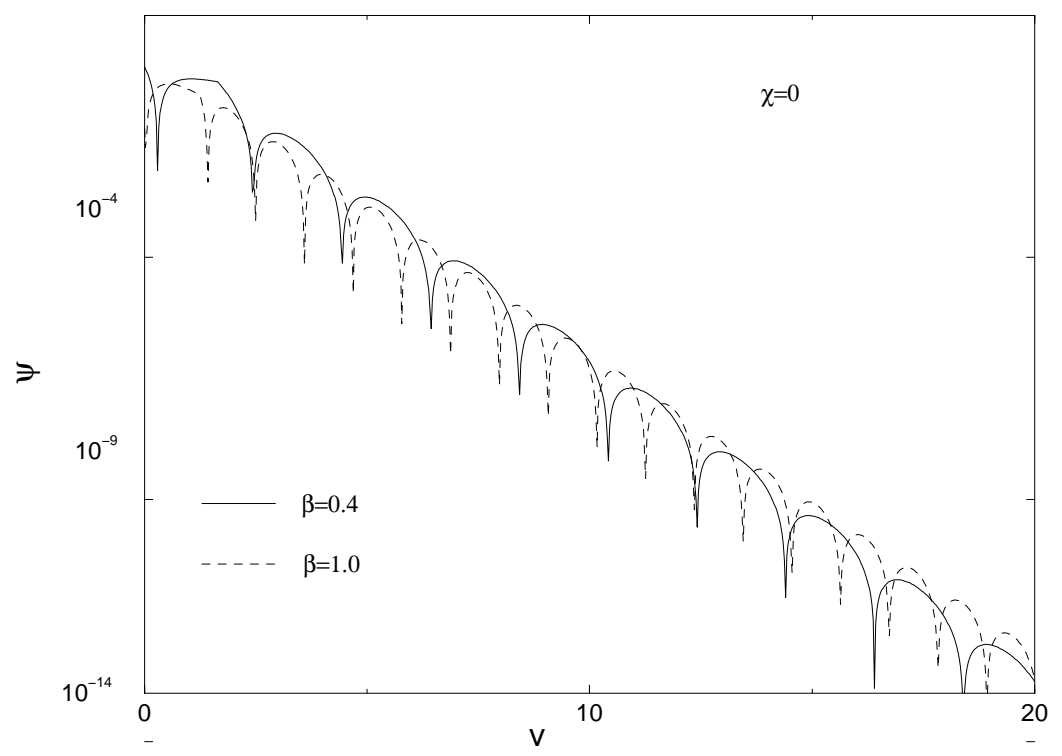

Figure 8: Behavior of field with the increase of $\beta$

negative mass. $\beta=1 / \sqrt{3}$ is associated with a black hole of zero mass.

\section{A. Positive mass $(0<\beta<1 / \sqrt{3})$}

We solve the scalar wave equation (16) where the potential $V$ has a shape similar to the $g=0$ case shown in figs.1ㄱ. This similarity yields wave propagation for different values of $\xi$ similar to that of the SAdS case. The results are illustrated in figs $10-13$ for fixed $\beta$. We found that for increasing $\chi$ (decreasing $\xi$ in the range $\xi \leq 1 / 6)$, both the real and imaginary parts of the quasinormal frequency outside the black hole increase. We thus see that outside the topological black hole with positive mass, if the field is more weakly non-minimally coupled to spacetime curvature, the thermal perturbation will settle down faster. Therefore, we qualitatively observe the same behaviour as that seen in black holes with genus $g=0$ and $g=1$ cases. 

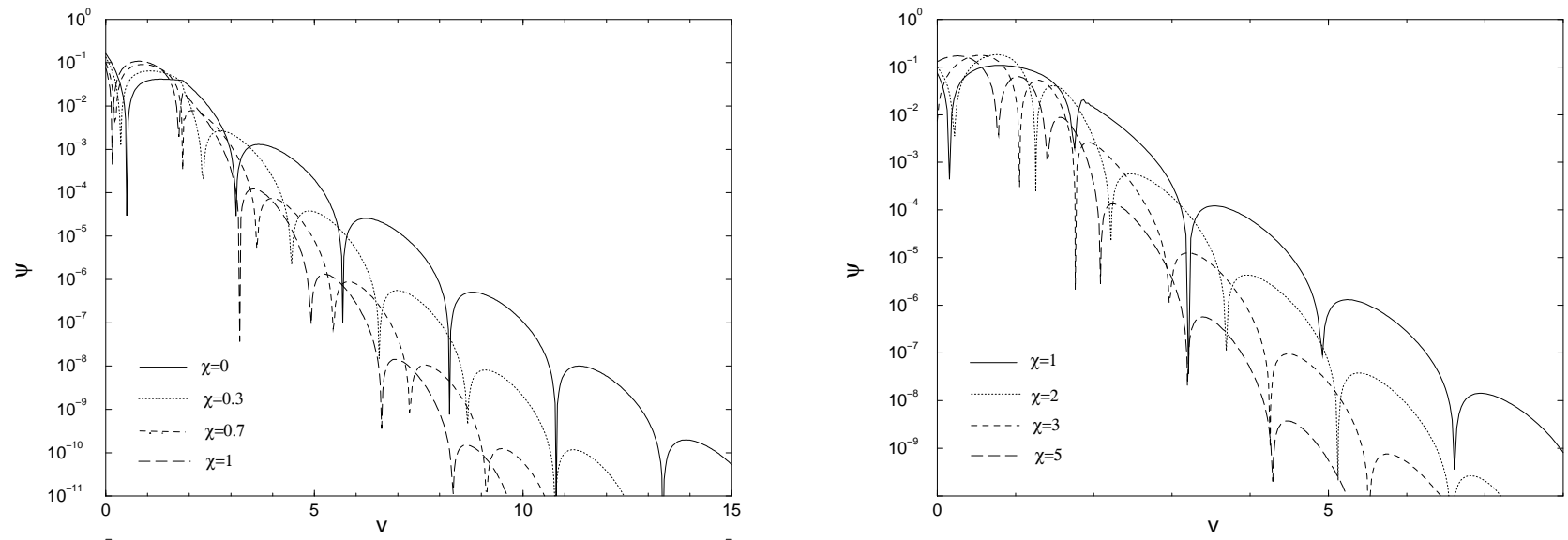

Figure 9: Falloff behavior for $\xi \leq 1 / 6$ in toroidal black holes

For $\xi>1 / 6$, the similar divergence of the potential to negative infinity as shown in fig.3 leads to amplification of the mode again.

Fig.14 illustrates the oscillation behaviour for various values of $\beta$. We see that the decay rate (the imaginary part $\tilde{\omega}_{I}$ of the quasinormal frequency) does not change much as $\beta$ increases. Using the relation (19), we see that this property corresponds to the decrease of both the real $\omega_{R}$ and imaginary $\omega_{I}$ parts of the quasinormal frequency with decreasing black hole size. The range of $\beta$ shown in fig.14 corresponds to intermediate-size black holes. Compared to intemediate Schwarzschild AdS holes, we find that falloff behaviour remains similar, while the field oscillates less rapidly. As $\beta \rightarrow 1 / \sqrt{3}$, the ringing frequency markedly decreases and finally disappears.

For large black holes ( $\beta$ very small), no explicit difference has been observed in wave propagation with different small $\beta$ in our scale. This behaviour is similar to $g=0$ case which corresponds to the increase of both real and imaginary parts of frequencies with the increase of the size of big black hole in the real time scale. 

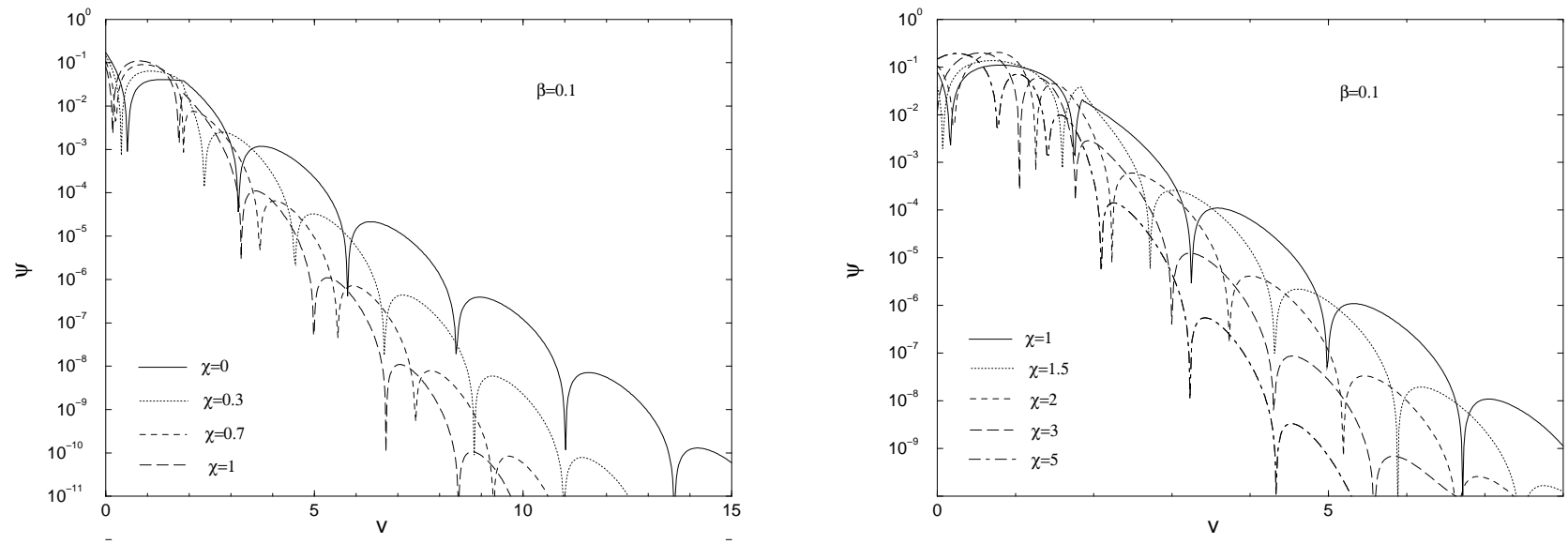

Figure 10: Falloff behavior for $\xi \leq 1 / 6$ in positive mass topology black holes when $\beta=0.1$

\section{B. Zero mass $(\beta=1 / \sqrt{3})$}

According to Eq.(13), when $\beta=1 / \sqrt{3}, V$ vanishes for a scalar field conformally coupled to curvature for the topological black hole with genus $g \geq 2$. As a result, the conformally invariant scalar field propagates freely in topological black hole spacetimes of $\beta=1 / \sqrt{3}$. The behaviour of the scalar wave conformally coupled to curvature is illustrated in fig.15 with $\chi=0$, which coincides with that shown in ref. [11]. The "blip" in the wave is due to the vanishing potential and the Dirichlet boundary condition we have taken is as explained in ref. [11].

It is interesting to study the $\xi$-dependence of wave function. For $\xi<1 / 6$, the potential deviates from zero and exhibits behaviour similar to that shown in fig.2 for the $g=0$ case with increasing of $\chi$ (decreasing $\xi$ ). The behaviour of the wave for $\xi<1 / 6$ is shown in fig. 15 for $\chi \neq 0$. We find that the falloff rate increases with the increasing of $\chi$, in agreement with the aforementioned behaviour. There is no oscillation of the wave appear here. 

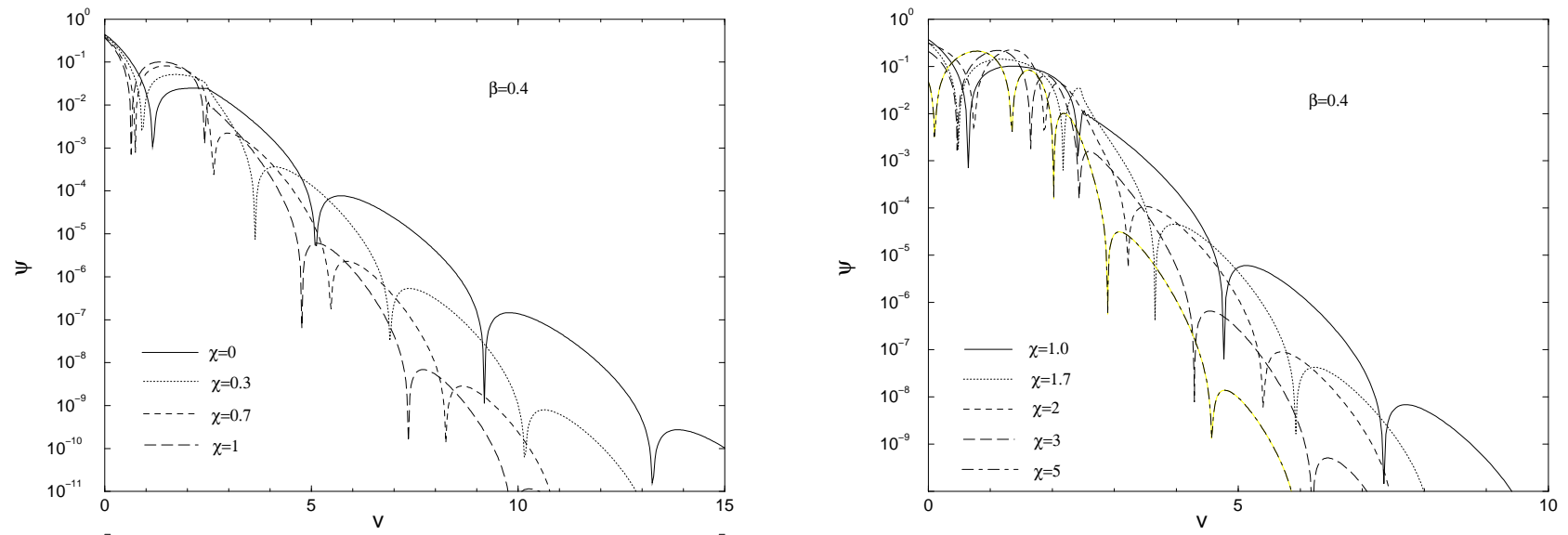

Figure 11: Falloff behavior for $\xi \leq 1 / 6$ in positive mass topology black holes when $\beta=0.4$

For $\xi>1 / 6$, the potential tends to negative infinity as shown in Fig. 3, and amplification appears again.

\section{Negative mass $(1 / \sqrt{3}<\beta \leq 1)$}

When $\beta>1 / \sqrt{3}$, the black hole mass is negative. This rather strange situation can be realized by the collapse of matter that violates the standard energy conditions under certain circumstances [20]. For $\xi=1 / 6$, the potential for $\beta>1 / \sqrt{3}$ outside the black hole becomes everywhere negative. This is quite different from the $g=0,1$ cases and also different from the positive mass higher genus black hole case. Some graphs of the potential for $\beta>1 / \sqrt{3}$ are shown in Fig.16. We see that larger values of $\beta$ lead to more negative values of the potential. Combing this property with the conjecture of ref. [13] in determining the sign of imaginary part of the frequency (eq.(2.22) in [13]),

$$
\int_{r_{+}}^{\infty} d r\left[f\left|\psi^{\prime}\right|^{2}+V|\psi|^{2}\right]=-\frac{|\omega|^{2}\left|\psi\left(r_{+}\right)\right|^{2}}{\omega_{I}}
$$



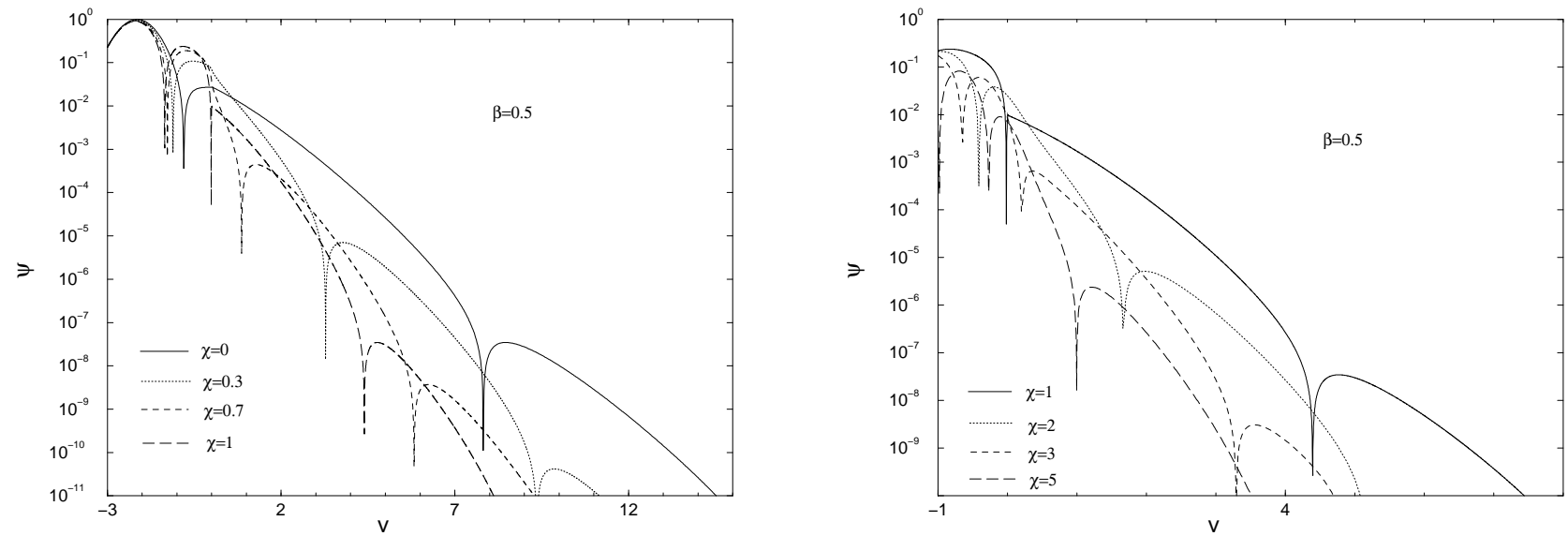

Figure 12: Falloff behavior for $\xi \leq 1 / 6$ in positive mass topology black holes when $\beta=0.5$

it might be possible to understand the amplification results shown in [11] and also in fig.18 below for large $\beta$ when $\xi=1 / 6(\chi=0)$. The negative potential for larger $\beta$ changes the sign of the left-hand-side of the above equation, thereby reversing the sign of $\omega_{I}$, yielding amplification.

When $\xi<1 / 6$, the potential becomes positive and tends to infinite at infinity. The $\xi$-dependence of the potential for $\xi<1 / 6$ has the same character as that shown in fig.2, which contributes to the same behaviour of the wave for increasing $\chi$ (decreasing $\xi$ ) in the range $\xi<1 / 6$. The scalar fields decays faster the nonminimal coupling to the spacetime curvature is weaker. This behaviour is shown in figs.17,18.

When $\xi>1 / 6(\chi<0)$, the potential goes to negative infinity for all $\beta$ as shown in Fig. 3. The wave outside the black hole experiences amplification instead of decay. This result is the same as for other black holes with $\xi>1 / 6$.

Comparing figs.17,18, we find that for fixed $\xi<1 / 6$, the wave decays slows 


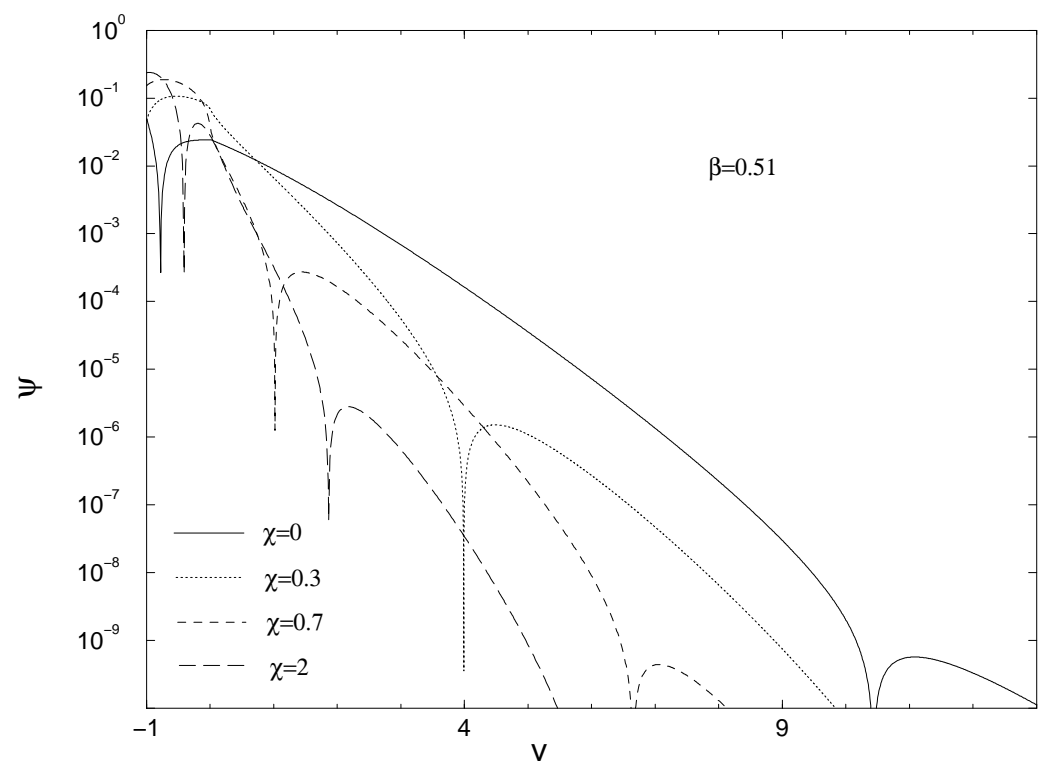

Figure 13: Falloff behavior for $\xi \leq 1 / 6$ in positive mass topology black holes when $\beta=0.51$

with increasing $\beta$. Using eq.(19), this corresponds to the fact that the imaginary part $\omega_{I}$ of thefrequency decreases with decreasing small black hole size, similar to the behaviour observed for small SAdS and positive-mass topological black holes. This agreement is due to the similar behaviour of the potential outside these various black holes.

\section{Conclusions and discussion}

We have studied the propagation of scalar waves non-minimally coupled to curvature in black hole backgrounds of different genus $g$. The coupling constant $\xi$ plays an important role and leads to a rich spectrum of wave evolution. Physically, non-minimal curvature coupling tends to erode falloff behaviour: for $\xi<1 / 6$, the 

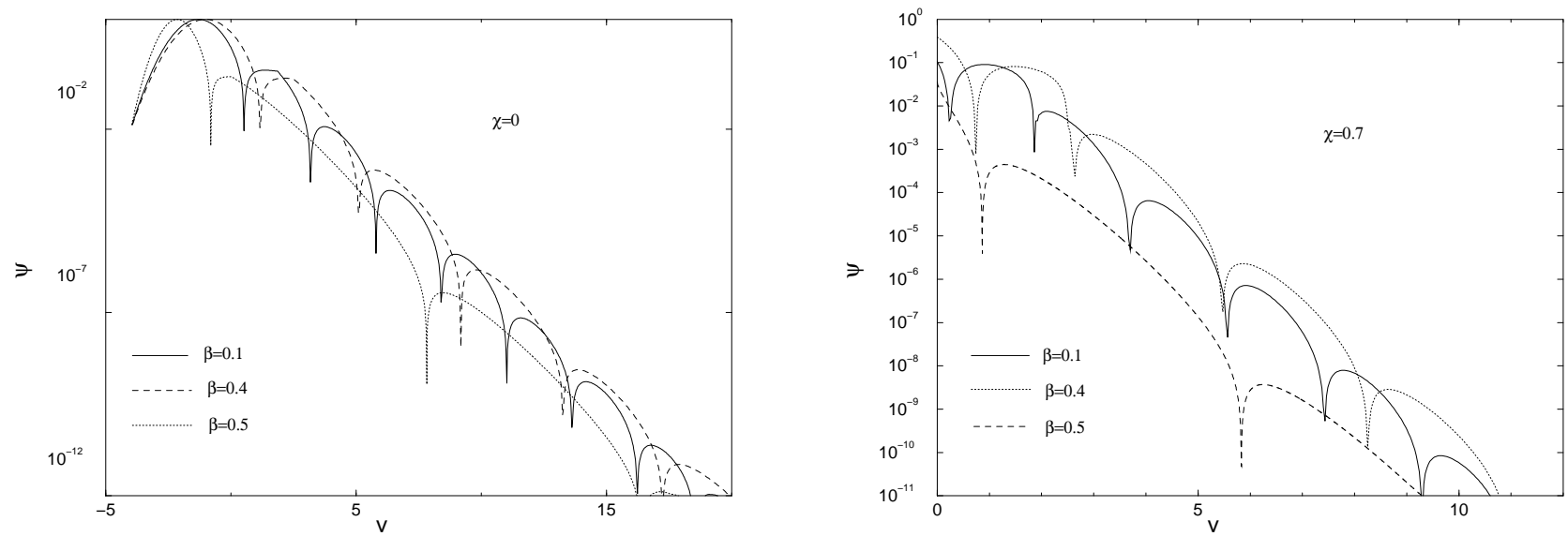

Figure 14: wave bahavior with the increase of $\beta$ in positive mass topological black holes

field decays monotonically with a decay constant that increases with decreasing $\xi$. This behaviour holds for all topological black holes with differing genus, and is a result of the qualitatively similar behaviour of their potentials. Our results differ from those obtained in de Sitter spacetime [10] where it was found that the decay constant increases with increasing $\xi$ for $\xi<1 / 6$. However in the de Sitter case the potential falls off exponentially at both the black hole and cosmological horizons for arbitrary $\xi$. This differs completely from the situation we are studying here with $\xi<1 / 6$, and in light of the observations of Ching et al [21] not surprising.

The waves outside the black hole experience oscillation for all positive mass black holes with different topologies. The oscillation frequency increases as $\xi$ decreases and $\xi<1 / 6$. For zero mass and negative mass higher genus black holes, the wave outside the black hole displays only decay without ringing.

For $\xi=1 / 6$, which corresponds conformally coupling to the curvature, our 


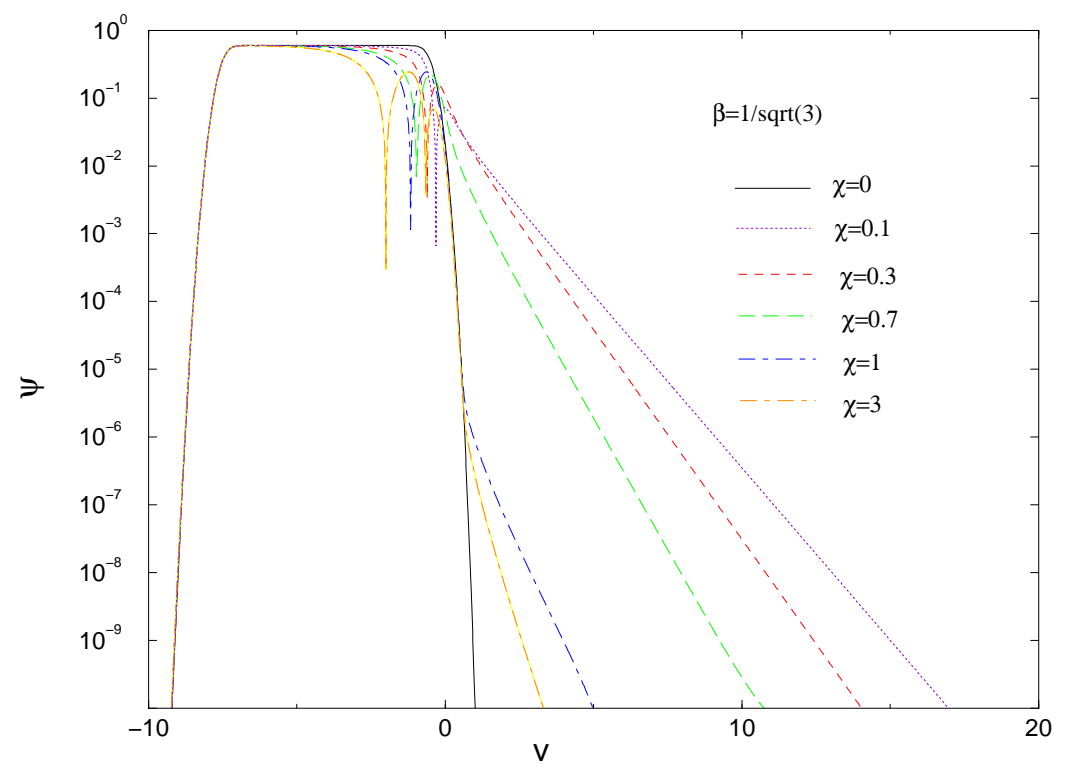

Figure 15: Falloff behavior for $\xi \leq 1 / 6$ in zero mass topology black holes

results coincide with those obtained in [11]. The wave amplification for negative mass topological black holes when $\beta$ is close to unity can be understood by using eq. (201).

For $\xi>1 / 6$, potentials tend to negative infinity for large distance outside topological black holes. Waves amplify instead of decay outside black holes of any genus. The amplitude of amplification is stronger than that found for negative mass topological hole with large $\beta$ at $\xi=1 / 6$. This is because the potential for $\xi>1 / 6$ case is more negative than that negative mass topological with big $\beta$ at $\xi=1 / 6$.

We have also explored the dependence of quasinormal ringing on $\beta$. Upon taking account of the coordinate rescaling in (111.12), the imaginary part of the quasinormal frequency decreases with $r_{+}$for small or intermediate size black holes, regardless of genus. However the real part $\omega_{R}$ of the quasinormal frequency 

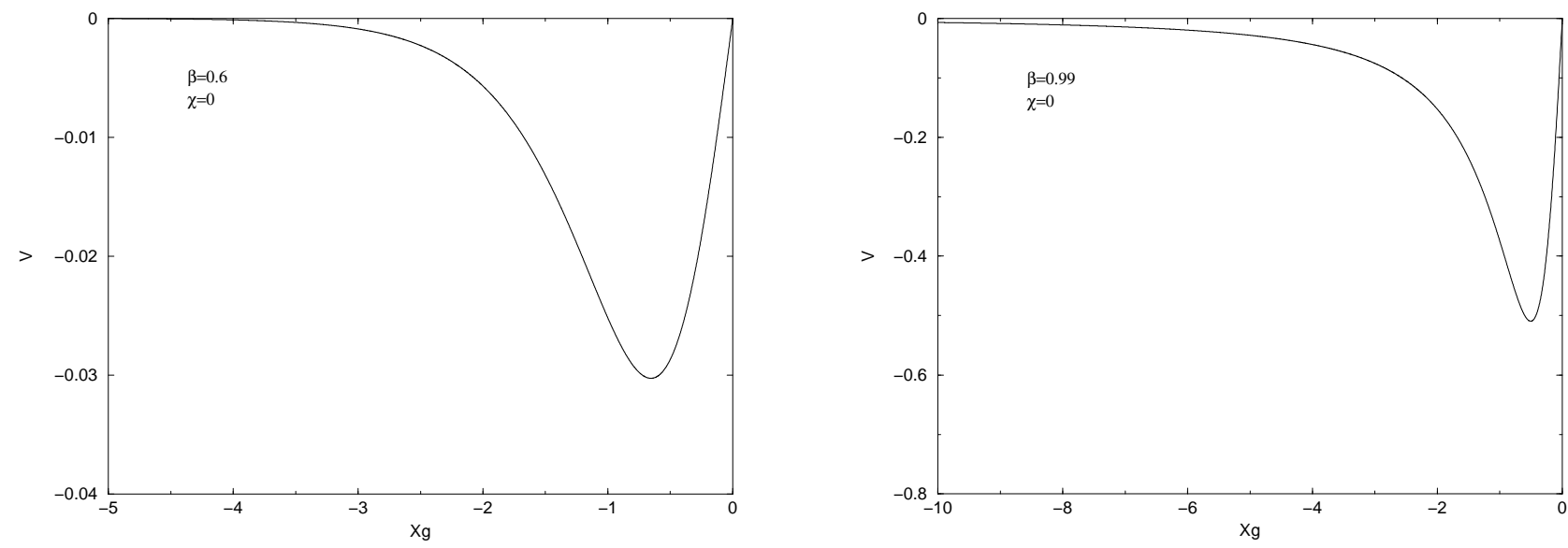

Figure 16: Potential behavior for negative mass topology black hole when $\xi=1 / 6$

has some topological dependence. For intermediate or small SAdS black holes, $\omega_{R}$ does not change with the black hole size, whereas for positive mass higher genus small black holes, $\omega_{R}$ decreases with decreasing black hole size. When the positive mass black hole is small enough $(\beta \rightarrow 1 / \sqrt{3})$, the wave oscillations disappear. For large black holes ( $\beta$ very small), both $\omega_{R}$, and $\omega_{I}$ increase with increasing black hole size and this property is the same for black holes with $g=0$ and $g \geq 2$ cases.

\subsubsection{ACKNOWLEDGMENT:}

This work was partically supported by Fundacão de Amparo à Pesquisa do Estado de São Paulo (FAPESP) and Conselho Nacional de Desenvolvimento Científico e Tecnológico (CNPQ). B. Wang would like to acknowledge helpful discussions on numerical problem with T. Osada and C. Molina and hospitality given by the Dept. of Physics at the University of Waterloo. The work of B. Wang was also supported by NNSF of China. 

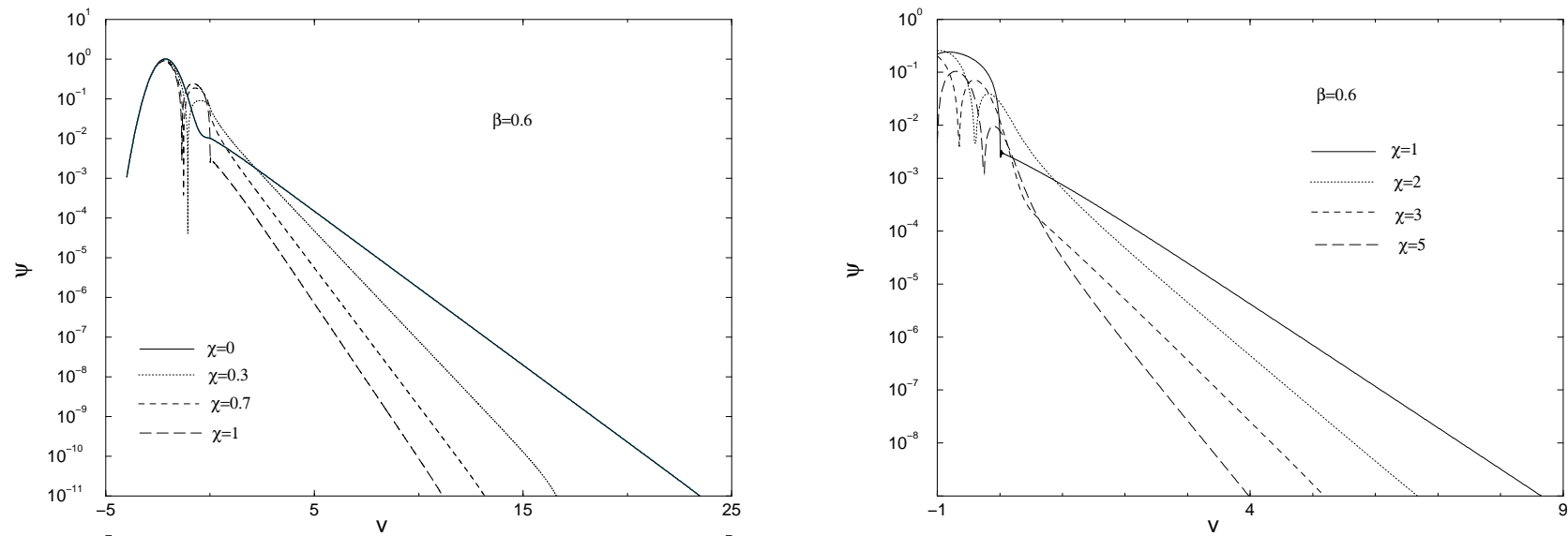

Figure 17: wave propagation in the negative mass topological black hole background with different $\xi$ when $\beta=0.6$

\section{References}

[1] K. D. Kokkotas, B. G. Schmidt, Living Rev. Rel. 2, 2 (1999), gr-qc/9909058.

[2] R. H. Price, Phys. Rev. D 5, 2419 (1972); 5, 2439 (1972).

[3] C. Gundlach, R. H. Price and J. Pullin, Phys. Rev. D 49, 883 (1994).

[4] J. Bicak, Gen. Relativ. Gravit. 3, 331 (1972).

[5] S. Hod, Phys. Rev. D 58, 104022 (1998); 61, 024033(2000); 61, 064018 $(2000)$.

[6] L. Barack and A. Ori, Phys. Rev. Lett. 82, 4388 (1999); Phys. Rev. D 60, 124005 (1999).

[7] N. Andersson and K. Glampedakis, Phys. Rev. Lett. 84, 4537 (2000). 


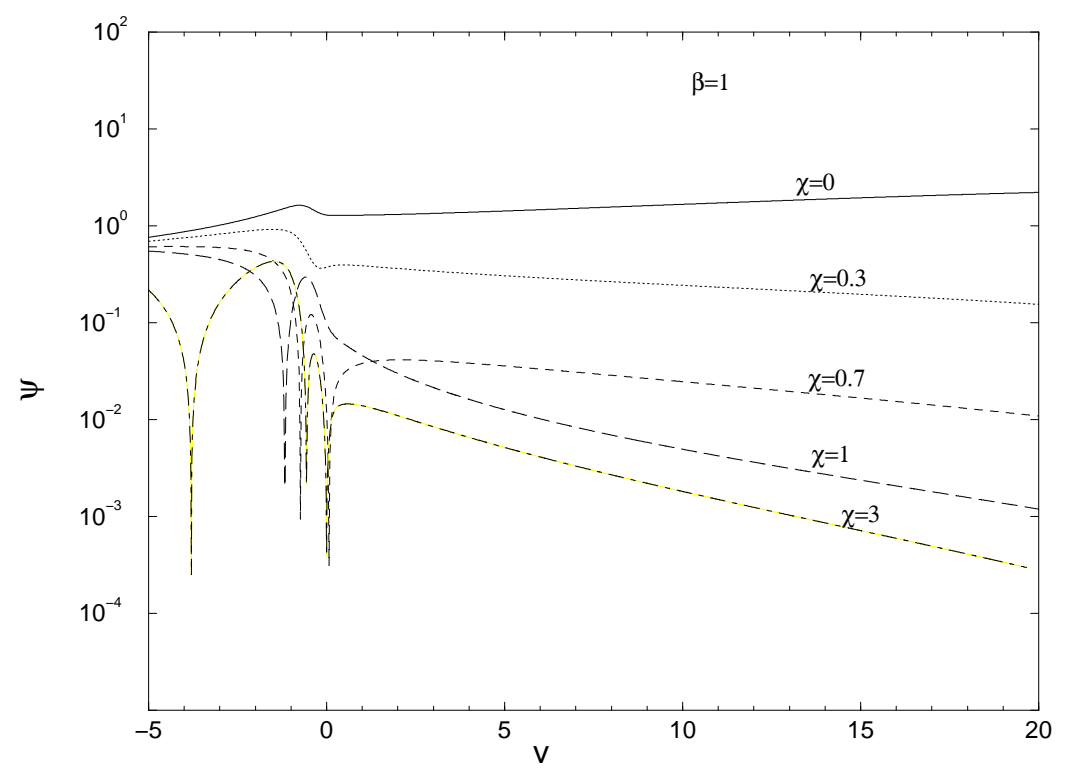

Figure 18: wave propagation in the negative mass topological black hole background with different $\xi$ when $\beta=1.0$

[8] P. R. Brady, C. M. Chambers, W. Krivan and P. Laguna, Phys. Rev. D 55, 7538 (1997).

[9] P. R. Brady, C. M. Chambers, W. G. Laarakkers and E. Poisson, Phys. Rev. D 60, 064003 (1999).

[10] W. G. Laarakkers and E. Poisson, gr-qc/0105016.

[11] J. S. F. Chan and R. B. Mann, Phys. Rev. D 59, 064025 (1999).

[12] J. S. F. Chan and R. B. Mann, Phys. Rev. D 55, 7546 (1997)

[13] G. T. Horowitz and V. E. Hubeny, Phys. Rev. D 62, 024027 (2000); G. T. Horowitz, Class. Quant. Grav. 17, 1107 (2000). 
[14] J. M. Zhu, B. Wang and E. Abdalla, Phys. Rev. D 63, 124004 (2001).

[15] B. Wang, C. Y. Lin and E. Abdalla, Phys. Lett. B 481, 79 (2000), hepth/0003295.

[16] B. Wang, C. Molina and E. Abdalla, Phys. Rev. D 63, 084001 (2001).

[17] V. Cardoso, J. P. S. Lemos, Phys. Rev. D 63, 124015 (2001); gr-qc/0105103.

[18] E. Winstanley, gr-qc/0106032.

[19] R. B. Mann, Nucl. Phys. B 516, 357 (1998).

[20] R. B. Mann, Class. Quant. Grav. 14, 2927 (1997).

[21] E. S. C. Ching, P. T. Leung, W. M. Suen and K. Young, Phys. Rev. D 52, 2118 (1995); Phys. Rev. Lett. 74, 2414 (1995). 